\title{
Can Intraprostatic Injection of OnabotulinumtoxinA be Beneficial to Treat Premature Ejaculation? Results of a Prospective Study
}

\author{
Taha A. Abdel-Meguid 1,2, MBBS, MSc, MD, Ahmed J. Alsayyad', FRCSC, \\ Abdulmalik M. Tayib ${ }^{1}$, FACHARTZ, Hasan M. Farsi ${ }^{1}$, FRCSC, Hisham A. Mosli' ${ }^{1}$, FRCSC, \\ Moataz Sait ${ }^{1}$, MBBS, Ahmed Abdelsalam ${ }^{1}$, MBBS, MSc, MD \\ 'Department of Urology, Faculty of Medicine, King Abdulaziz University, Jeddah, Saudi Arabia; \\ ${ }^{2}$ Department of Urology, Minia University, Minia, Egypt.
}

\author{
Correspondence \\ Prof. Taha A. Abdel-Meguid \\ P.O. Box 80215, Jeddah 21589, Saudi Arabia \\ e.M: tahaaboalmagd@yahoo.com \\ Submission: 20 August 2017 \\ Accepted: $\quad 04$ September 2017

\section{Citation} \\ Abdel-Meguid TA, Alsayyad AJ, Tayib AM, \\ Farsi HM, Mosli HA, Sait M, Abdelsalam A. Can \\ intraprostatic injection of OnabotulinumtoxinA \\ be beneficial to treat premature ejaculation? \\ Results of a prospective study. JKAU Med Sci \\ 2017; 24 (4): 1-7. DOI: 10.4197/Med. 24.4.1
}

\begin{abstract}
We prospectively evaluated efficacy and adverse effects of intraprostatic injections of onabotulinumtoxinA to treat premature ejaculation. Twentyfour men $\geq 19$ years-old with premature ejaculation for $\geq 6$ months and intravaginal ejaculation latency time $\leq 2$ minutes underwent transurethral intraprostatic injections of onabotulinumtoxinA (100 U). Primary endpoint was change of intravaginal ejaculation latency time at 3-months. Secondary endpoints included changes in premature ejaculation profile and patientreported global impression of change (PGI). Mean baseline ejaculation latency time has significantly increased at 1-, 3- and 6-months, respectively. In premature ejaculation profile "perceived control over ejaculation", significant improvement was reported at 3-months, while non-significant changes were reported at 1- and 6-months. Patients reported non-significant changes of "personal distress related to ejaculation" and "interpersonal difficulty related to ejaculation". Only $8.3 \%, 12.5 \%$ and $12.5 \%$ of men reported "better" at 1-, 3-and 6-months, respectively, while all other patients reported "no change" or "slightly better" in patient-reported global impression of change. No serious adverse effects were observed. Improvements of intravaginal ejaculation latency time were not clinically meaningful, as most men reported "no change" or "slightly better" in patient-reported global impression of change. These marginal improvements did not support using onabotulinumtoxinA intraprostatic injections to remedy premature ejaculation.
\end{abstract}

\section{Keywords}

Intravaginal ejaculation latency time; OnabotulinumtoxinA; Rapid ejaculation; Sexual dysfunction; Treatment

\section{Introduction}

remature ejaculation $(\mathrm{PE})$ is the most common sexual dysfunction in males and has been reported in $5 \%$ to $40 \%$ of sexually active men according to their age. Premature ejaculation is more prevalent in sexually naive males, particularly adolescents and young adults ${ }^{[1]}$.

Although the pathophysiology of PE is still not completely understood, PE has been suggested to have both somatic and psychological basis, and it 
was regarded also to be highly neurobiologically determined ${ }^{1-3]}$. Pelvic plexus branches, integrating sympathetic and parasympathetic fibers, innervate the epididymis, vasa deferentia, seminal vesicles, prostate, bladder neck and urethra. The reflex of ejaculation encompasses sensory receptors, afferents, cerebral sensory and motor areas, spinal cord motor centers and efferents $s^{[1-3]}$.

OnabotulinumtoxinA (onaBoNT-A) is a potent neurotoxin, which mechanism of action has been traditionally thought to be mediated only via prolonged blockade of presynaptic release of acetylcholine at the neuromuscular junctions leading to chemical denervation and muscle paralysis ${ }^{[4]}$. More recent data, however, support the conception that onaBoNT-A acts on both sensory and motor pathways ${ }^{[4,5]}$. OnabotulinumtoxinA injections has been successfully applied in an increasing number of urologic disorders in both adults and children ${ }^{[6-9]}$. The effects of onaBoNT-A injections into the prostate have been studied and were considered to be safe since local or systemic side effects were rare $^{[7-9]}$. Injecting onaBoNT-A into bulbospongiosus and ischiocavernosus muscles has been reported to have a beneficial effect in extending the ejaculatory latency by suppressing their stereotyped rhythmic contractions ${ }^{[10,11]}$.

We hypothesized that chemical denervation of the prostateand ejaculatoryducts by intraprostaticinjection of onaBoNT-A may suppress their contractions during emission, and consequently delay the ejaculation. The objective of our study was to prospectively evaluate the efficacy and adverse effects (AEs) of intraprostatic injections of onaBoNT-A in treatment of PE.

\section{Methods}

\section{Study Design and Setting}

The current cohort study was prospectively conducted at our institution between October 2010 and June 2015. The study was approved by our institutional Ethics Committee and each patient provided an informed consent.

\section{Inclusion Criteria}

We included adult men $\geq 19$ years-of-age, who were in stable marital relationships for at least the last 6 months, fulfilled the Diagnostic and Statistical Manual of Mental Disorders (4th edition, text revision) (DSM-IV-TR) criteria for $P E$ for $\geq 6$ months ${ }^{[12]}$, reported intravaginal ejaculation latency time (IELT) of $\leq 2$ minutes in $\geq 75 \%$ of coital attempts at a 4-week baseline assessment period, and indicated at least moderate PE-related personal distress or interpersonal difficulty.

\section{Exclusion Criteria}

History of serious medical, psychiatric or mental disorders, erectile or other sexual dysfunctions, partner's sexual dysfunction, serious marital relational problems, very low intercourse frequency and patients with urinary tract infections were excluded.

\section{Evaluation}

The tools used for patient's evaluation included stopwatch-measured ILETS, and patient-reported outcome measures ${ }^{[13]}$ combining premature ejaculation profile (PEP) and global impression of change (PGI) questionnaires at different study time points (Table 1). Intravaginal ejaculation latency time was measured by a stopwatch held by the partner as the time elapsing between starting vaginal penetration and starting intravaginal ejaculation. Premature ejaculation profile is a validated self-reported 4-domain 4-question questionnaire. Each question scales $0-4$ points with a total score of 0-16 points. Although PEP lacks validated cutoff scores, higher scores indicate better ejaculatory functions. PGI questionnaire describes the overall PE condition compared to baseline in a seven-point scale ranging from -3 to +3 points ("much worse" to "much better", respectively $)^{[13]}$.

The patients were initially evaluated at a 4-week baseline period as they were required to report the IELT of their coital attempts as measured by stopwatch and to complete PEP questionnaire as well. Follow up assessment was done at 1-, 3- and 6-month postinjections to evaluate IELT, PEP, and PGI. Urine analysis and urine culture were done pre-injections, 1-months post injections, and thereafter as required. The patients were instructed not to receive any other therapy for PE ahead of the baseline period and throughout study duration.

\section{Outcome Measurements}

The primary endpoint was change of IELT at 3-month follow up, as compared to baseline. Secondary endpoints included changes in PEP and PGI at different 
Table 1. Premature ejaculation profile and patient-reported global impression of change in premature ejaculation $\left[{ }^{[3]}\right.$.

\begin{tabular}{|c|l|l|l|}
\hline Measure & \multicolumn{1}{|c|}{ Domain } & \multicolumn{1}{|c|}{ Question } & \multicolumn{1}{c|}{ Score } \\
\hline \multirow{2}{*}{ PEP } & Perceived control over & "Over the past month, was your control over ejaculation & $0:$ Very poor \\
& ejaculation & during sexual intercourse:" & 1: Poor \\
& Satisfaction with sexual & "Over the past month, was your & 2: Fair \\
& intercourse & satisfaction with sexual intercourse:" & 3: Good \\
& & & $4:$ Very good \\
\cline { 2 - 4 } & Personal distress related to & "How distressed are you by how fast you ejaculated during & $0:$ Extremely \\
& ejaculation & sexual intercourse?" & 1: Quite a bit \\
\cline { 2 - 4 } & Interpersonal difficulty related & "To what extent does how fast you ejaculated during sexual & 2: Moderately \\
& to ejaculation & intercourse cause difficulty in your relationship with your & 3: Alittle bit \\
& & partner?" & 4: Not at all \\
\hline \multirow{2}{*}{ PGI } & Compared to prior to the study, would you describe your & $-3:$ Much worse \\
& & premature ejaculation condition as: & $-2:$ Worse \\
& & & $-1:$ Slightly worse \\
& & & $0:$ No change \\
& & & 1: Slightly better \\
& & & 2: Better \\
& & & 3: Much better \\
\hline
\end{tabular}

follow up time points. Local and systemic AEs were also reported.

\section{Procedure}

The injections were performed transurethrally under general or regional anesthesia while the patient was in lithotomy position and as a day care setting. A total dose of $100 \mathrm{U}$ of onaBoNT-A (BOTOX ${ }^{\circledR}$, Allergan, Inc., Irvine, CA USA) was reconstituted in $4 \mathrm{ml}$ of normal saline and divided into four fractions ( $1 \mathrm{ml}, 25 \mathrm{U}$ each). Prostatic tissues surrounding the ejaculatory ducts were aimed for injections using 23-gauge cystoscopic needle. Three intraprostatic injections were deeply allocated throughout floor of prostatic urethra, sparing $5 \mathrm{~mm}$ distance to the vicinity of bladder neck. A fourth most distal injection was allotted to the verumontanum close to ejaculatory ducts openings (Fig. 1).

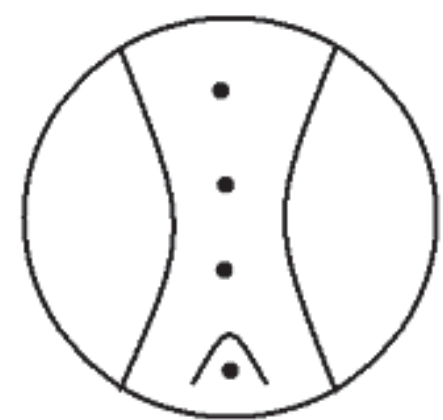

Figure 1. Sites of intraprostatic injections of onabotulinumtoxinA in floor of prostatic urethra.

\section{Statistical Analysis}

GraphPad Prism for Windows, version 6.0 (GraphPad Software Inc., La Jolla, CA) was used for data analysis. Descriptive statistics were expressed as frequencies, proportions, mean (SD), and 95\% confidence interval $(\mathrm{Cl})$. Differences over time among paired continuous data were analyzed using repeated measures ANOVA followed by Dunnett's test for correction of multiple comparisons. Double-sided $p<0.05$ was considered as significant.

\section{Results}

The study included 24 men with a mean age of 34.9 (8.9) yrs and mean IELT of 62.7 (25.9) seconds at baseline. The mean IELT has significantly increased from baseline to 79.1 (32.7), 89.0 (36.1) and 85.7 (35.4) seconds at 1-, 3and 6-months, respectively $(26.2 \%, 41.9 \%$, and $36.7 \%$ increase; $p<0.0001$ each). In PEP questionnaire, the score of "perceived control over ejaculation" has shown significant improvement at 3-months $(p=0.045)$, while at 1 - and 6-months non-significant changes were reported. Additionally, the PEP data demonstrated significant changes of "satisfaction with sexual intercourse" but non-significant changes of "personal distress related to ejaculation" and "interpersonal difficulty related to ejaculation" at all follow up time points (Table 2). As for PGl, while only 2 (8.3\%), 3 (12.5\%), and $3(12.5 \%)$ men reported "better" at 1-, 3and 6-months, respectively, all other patients reported 
Table 2. Outcomes of stopwatch-measured IELT, PEP and PGI at follow up time points.

\begin{tabular}{|c|c|c|c|c|}
\hline Measure & Baseline & 1-mo & 3-m & 6-mo \\
\hline $\begin{array}{l}\text { IELT (Sec) } \\
\text { Mean (SD) } \\
\text { MD }(95 \% \text { Cl) } \\
\% \text { change } \\
\text { P }\end{array}$ & $\begin{array}{c}62.7(25.9) \\
\text { NA } \\
\text { NA } \\
\text { NA }\end{array}$ & $\begin{array}{c}79.1(32.7) \\
16.4(12.9,19.9) \\
26.2 \% \\
0.0001>\end{array}$ & $\begin{array}{c}89.0(36.1) \\
26.3(20.9,31.7) \\
41.9 \% \\
0.0001>\end{array}$ & $\begin{array}{c}85.7(35.4) \\
23.0(18.2,27.9) \\
36.7 \% \\
0.0001>\end{array}$ \\
\hline $\begin{array}{l}\text { PEP (Points) } \\
\text { Perceived control over ejaculation }\end{array}$ & $\begin{array}{l}\text { Mean (SD) } \\
1.29(0.86)\end{array}$ & $\begin{array}{c}\text { MD }(95 \% \mathrm{Cl}), \mathbf{p} \\
0.29(-0.06,0.65) \\
P=0.1223\end{array}$ & $\begin{array}{c}\mathrm{MD}(95 \% \mathrm{CI}), \mathbf{p} \\
0.33(0.006,0.60) \\
p=0.0450\end{array}$ & $\begin{array}{c}\mathrm{MD}(95 \% \mathrm{Cl}), \mathbf{p} \\
0.21(-0.09,0.51) \\
p=0.2238\end{array}$ \\
\hline Satisfaction with sexual intercourse & $1.96(1.08)$ & $\begin{array}{c}0.29(0.01,0.57) \\
p=0.0418\end{array}$ & $\begin{array}{c}0.50(0.16,0.84) \\
p=0.0032\end{array}$ & $\begin{array}{c}0.42(0.02,0.82) \\
p=0.0388\end{array}$ \\
\hline Personal distress related to ejaculation & $1.63(0.65)$ & $\begin{array}{c}0.21(-0.005,0.42) \\
p=0.0560\end{array}$ & $\begin{array}{c}0.17(-0.19,0.53) \\
p=0.5231\end{array}$ & $\begin{array}{c}0.042(-0.31,0.40) \\
p=0.9822\end{array}$ \\
\hline Interpersonal difficulty related to ejaculation & $1.83(0.48)$ & $\begin{array}{c}0.21(-0.16,0.58) \\
p=0.3715\end{array}$ & $\begin{array}{c}0.25(-0.16,0.66) \\
p=0.3064\end{array}$ & $\begin{array}{c}0.29(-0.094,0.68) \\
p=0.1664\end{array}$ \\
\hline $\begin{array}{l}\text { PGI, } \mathbf{n} \text { (\%) } \\
\text { No change } \\
\text { Slightly better } \\
\text { Better }\end{array}$ & NA & $\begin{array}{c}13(54.2 \%) \\
10(41.7 \%) \\
2(8.3 \%)\end{array}$ & $\begin{array}{l}11(45.8 \%) \\
10(41.7 \%) \\
3(12.5 \%)\end{array}$ & $\begin{array}{l}12(50 \%) \\
9(37.5 \%) \\
3(12.5 \%)\end{array}$ \\
\hline
\end{tabular}

(IELT: Intravaginal ejaculation latency time; PEP: Premature ejaculation profile; PGI: Patient-reported global impression of change; MD: Mean difference; SD: Standard deviation; Cl: Confidence interval).

"no change" or "slightly better". Table 2 demonstrates the outcomes of PEP and PGI at follow up time points. No serious local or systemic AEs were observed. All patients demonstrated microscopic hematuria which has resolved spontaneously within one week. Transient dysuria was reported in $9(37.5 \%)$ patients. No other ejaculatory disorders, changed erectile function, stress urinary incontinence, UTI, or sepsis were reported.

\section{Discussion}

There are several current therapeutic options to treat PE with variable efficacy and considerable drawbacks. Many well-designed clinical studies on selective serotonin reuptake inhibitors (SSRIs) and antidepressants support the efficacy of dapoxetine, paroxetine, sertraline, fluoxetine and clomipramine for treatment of $\mathrm{PE}^{[14-16]}$. Dapoxetine is a short-acting effective well-tolerated SSRI that can be used as 'ondemand' treatment for PE. It is currently the only drug approved for PE treatment ${ }^{[14-16]}$. A meta-analysis of studies of pharmacological treatments has found that paroxetine demonstrates the strongest delay of ejaculation with 8.8 fold mean IELT increase ${ }^{[17]}$. While drug treatments of PE have been demonstrated to be effective and reliable, their efficacy is often limited to the time of drug usage. In fact, the majority of men who achieved good ejaculatory control has experienced PE again after cessation of the medication ${ }^{[14-17]}$.
Antidepressant drugs may also considerably aggravate erectile dysfunction and they are strongly unadvisable for men suffering concomitant $\mathrm{PE}$ and erectile dysfunction ${ }^{[17]}$. Additionally, chronic SSRI treatment has been reported to cause deleterious effects on spermatogenesis and spermatozoa ${ }^{[19]}$. Topical anesthetics ${ }^{[20]}$ and herbal products ${ }^{[21]}$ have also been used in premature ejaculation of neurobiological origin due to penile hypersensitivity; although their use is not diffuse.

Based on the premises that PE has an underlying neurobiological pathophysiological basis, it has been suggested that penile and other sensory receptors play a major role in conveying sexual information to the cerebral cortex which controls and commands the cascade of events of ejaculatory reflex, and that spinal cord reflex plays a primary role in ejaculation ${ }^{[1-3]}$. Thus, any station in the route from penis to cerebral cortex (including the prostate) may be integrated in the process of PE. Nevertheless, the epididymis, vasa deferentia, seminal vesicles, prostate and bladder neck are the actual 'executives' of this cascade by emission of seminal fluid ${ }^{[1-3]}$. There are several additional indicators suggesting that the prostate and its innervations play an essential role in the process of ejaculation. For instance, alpha blockers - mainly tamsulosin - have been reported to induce ejaculation failure ${ }^{[22]}$. Additionally, electroejaculation has been used effectively for sperm 
retrieval in men with spinal cord injury ${ }^{[23]}$. The strong association of chronic prostatitis and rapid ejaculation further suggests a prostatic role in $\mathrm{PE}^{[24,25]}$.

In view of drawbacks of the existing therapies for $P E$, injection of botulinum-A toxin was suggested as a promising alternative PE treatment ${ }^{[10,11,26,27]}$. The relatively prolonged chemical denervation reported with onaBoNT-A injection may render PE men capable to spontaneously engage in sexual activity without requiring oral medications or applying topical treatment ${ }^{[28]}$. In two patent applications, Gaxiola et al. ${ }^{[26]}$ have proposed treating $\mathrm{PE}$ and prolonging ejaculation latency by injecting onaBoNT-A into the penis; or into the frenulum, prepuce, or glans penis ${ }^{[27]}$. Nevertheless, the experiments based on those patent proposals have not yet been published in any peer-reviewed publication. Serefoglu et al. ${ }^{[10]}$ have hypothesized that inhibition of the stereo-typed rhythmic contractions of bulbospongiosus and ischiocavernosus muscles by injecting onaBoNT-A into these muscles may have a favorable effect in treatment of PE. Those investigators have reported significant prolongation of ejaculatory latency in rats, without affecting their ability to engage in sexual activity or achieving ejaculation ${ }^{[11]}$. The hypothesis of Serefoglu et al., however, lacks a solid physiological foundation as the emission phase of the ejaculatory process precedes the rhythmic contractions of bulbospongiosus and ischiocavernosus muscles ${ }^{[1-3]}$.

Our investigation was based on the premise that chemical denervation of the prostate and ejaculatory ducts by intraprostatic injection of onaBoNT-A might suppress the emission of seminal fluid, and consequently delay the ejaculation. Although the present study reported statistically significant changes of IELT at all follow up time points after intraprostatic injections of onaBoNT-A, these improvements were not translated into clinically meaningful changes. As for "perceived control over ejaculation", the studied men reported barely modest improvement at 3-months, but non-significant changes at 1- and 6-months' time points. Only $8.3 \%, 12.5 \%$ and $12.5 \%$ of men reported "better" in PGI questionnaire at 1-, 3- and 6-months, respectively. Nevertheless, all other men have reported "no change" or "slightly better", and none of the men have reported "much better" at any follow up time points. These PGI findings further elucidate the marginal improvement of PE condition after intraprostatic onaBoNT-A injections. Considering the small improvement of PE condition in our study (in addition to invasiveness and cost of the procedure), this treatment did not prove itself compelling to be recommended for managment of $\mathrm{PE}$.

\section{Conclusion}

Our study demonstrated statistical improvements of IELT after intraprostatic injections of onaBoNT-A, that were not clinically meaningful as the majority of men reported "no change" or "slightly better" in PGI questionnaire. Although safe, our findings of marginal improvements did not support using this procedure to remedy $\mathrm{PE}$.

\section{Acknowledgements}

This project was funded by the Deanship of Scientific Research, King Abdulaziz University, Jeddah, Saudi Arabia, under grant no. (322/140/1431).

The authors, therefore, acknowledge with thanks Deanship of Scientific Research technical and financial support.

\section{Conflict of Interests}

None of the authors has any conflict of interests with any company.

The funding agency is a non-profit governmental agency, concerned with funding and supporting qualified peer-reviewed research projects. The funding agency has no influence on the results of the study.

\author{
Abbreviations and Acronyms \\ $\mathrm{Cl}=$ Confidence interval \\ IELT = Intravaginal ejaculation latency time \\ $\mathrm{MD}=$ Mean difference \\ OnaBoNT-A = OnabotulinumtoxinA \\ $\mathrm{PE}=$ Premature ejaculation \\ PEP = Premature ejaculation profile \\ $P G I=$ Patient-reported global impression of change \\ $\mathrm{SD}=$ Standard deviation \\ SSRIs = Selective serotonin reuptake inhibitors
}

\section{Reference}

[1] Jannini EA, Lenzi A. Ejaculatory disorders: epidemiology and current approaches to definition, classification and subtyping. World J Urol 2005; 23(2): 68-75.

[2] Kandeel FR, Koussa VK, Swerdloff RS. Male sexual function and its disorders: physiology, pathophysiology, clinical investigation, and treatment. Endocr Rev 2001; 22(3): 342-88.17 
[3] Jannini EA, Ciocca G, Limoncin E, Mollaioli D, Di Sante S, Gianfrilli D, Lombardo F, Lenzi A. Premature ejaculation: old story, new insights. Fertil Steril 2015; 104(5): 1061 1073.

[4] Chapple C, Patel A. Botulinum toxin--new mechanisms, new therapeutic directions? Eur Urol 2006; 49(4): 606608.

[5] Chuang YC, Yoshimura N, Huang CC, Wu M, Chiang PH, Chancellor MB. Intraprostatic botulinum toxin a injection inhibits cyclooxygenase-2 expression and suppresses prostatic pain on capsaicin induced prostatitis model in rat. J Urol 2008; 180(2): 742-748.

[6] Abdel-Meguid TA. Botulinum toxin-A injections into neurogenic overactive bladder-to include or exclude the trigone? A prospective randomized controlled trial. J Urol 2010; 184(6): 2423-2428.

[7] Kuo YC, Kuo HC. Botulinum toxin injection for lower urinary tract dysfunction. Int J Urol 2013; 20(1): 40-55.

[8] Chuang YC, Tu CH, Huang CC, Lin HJ, Chiang PH, Yoshimura $\mathrm{N}$, Chancellor MB. Intraprostatic injection of botulinum toxin type-A relieves bladder outlet obstruction in human and induces prostate apoptosis in dogs. BMC Urol 2006; 6 : 12.

[9] Oeconomou A, Madersbacher H, Kiss G, Berger TJ, Melekos $M$, Rehder P. Is botulinum neurotoxin type A (BoNT-A) a novel therapy for lower urinary tract symptoms due to benign prostatic enlargement? A review of the literature. Eur Urol 2008; 54(4): 765-775.

[10] Serefoglu EC, Silay MS. Botulinum toxin-A injection may be beneficial in the treatment of life-long premature ejaculation. Med Hypotheses 2010; 74(1): 83-84.

[11] Serefoglu EC, Hawley WR, Lasker GF, Grissom EM, Mandava SH, Sikka SC, Dohanich GP, Hellstrom WJ. Effect of botulinum-A toxin injection into bulbospongiosus muscle on ejaculation latency in male rats. J Sex Med 2014; 11(7): 1657-1663.

[12] American Psychiatric Association. Diagnostic and Statistical Manual of Mental Disorders. 4th edition, Text Revision (DSM-IV-TR). American Psychiatric Association. Washington, DC; 2000.

[13] Patrick DL, Giuliano F, Ho KF, Gagnon DD, McNulty $P$, Rothman M. The Premature Ejaculation Profile: validation of self-reported outcome measures for research and practice. BJU Int 2009; 103(3): 358-64.

[14] Castiglione F, Albersen M, Hedlund P, Gratzke C, Salonia A, Giuliano F. Current pharmacological management of premature ejaculation: A systematic review and metaanalysis. Eur Urol 2016; 69(5): 904-916.

[15] Althof SE, McMahon CG. Contemporary management of disorders of male orgasm and ejaculation. Urology 2016; 93: 9-21.

[16] Gur S, Sikka SC. The characterization, current medications, and promising therapeutics targets for premature ejaculation. Andrology 2015; 3(3): 424-442.
[17] Waldinger MD, Zwinderman AH, Schweitzer DH, Olivier B. Relevance of methodological design for the interpretation of efficacy of drug treatment of premature ejaculation: a systematic review and meta-analysis. Int J Impot Res 2004; 16(4): 369-381.

[18] Lasker GF, Halis F, Gokce A. Selective serotonin reuptake inhibitors for premature ejaculation: review of erectile and ejaculatory side effects. Curr Drug Saf 2014; 9(2): 118-126.

[19] Koyuncu H, Serefoglu EC, Ozdemir AT, Hellstrom WJ. Deleterious effects of selective serotonin reuptake inhibitor treatment on semen parameters in patients with lifelong premature ejaculation. Int J Impot Res 2012; 24(5): 171173.

[20] Berkovitch M, Keresteci AG, Koren G. Efficacy of prilocainelidocaine cream in the treatment of premature ejaculation. J Urol 1995; 154(4): 1360-1361.

[21] Choi HK, Jung GW, Moon KH, Xin ZC, Choi YD, Lee WH, Rha $\mathrm{KH}$, Choi YJ, Kim DK. Clinical study of SS-cream in patients with lifelong premature ejaculation. Urology 2000; 55(2): 257-261.

[22] van Dijk MM, de la Rosette JJ, Michel MC. Effects of alpha(1)adrenoceptor antagonists on male sexual function. Drugs 2006; 66(3): 287-301.

[23] Sønksen J, Ohl DA. Penile vibratory stimulation and electroejaculation in the treatment of ejaculatory dysfunction. Int J Androl 2002; 25(6): 324-32.

[24] Liang CZ, Hao ZY, Li HJ, Wang ZP, Xing JP, Hu WL, Zhang TF, Ge WW, Zhang XS, Zhou J, Li Y, Zhou ZX, Tang ZG, Tai $S$. Prevalence of premature ejaculation and its correlation with chronic prostatitis in Chinese men. Urology 2010; 76(4): 962-966.

[25] Screponi E, Carosa E, Di Stasi SM, Pepe M, Carruba G, Jannini EA. Prevalence of chronic prostatitis in men with premature ejaculation. Urology 2001; 58(2): 198-202.

[26] Gaxiola GM, Aguilar IE, Paz GP, inventors; Allergan, Inc. (Irvine, CA), assignee. Method for treating premature ejaculation with a botulinum neurotoxin. US 8329193 B2. 2012.

[27] Gaxiola GM, Aguilar IE, Paz GP, inventors; Allergan, Inc. (Irvine, CA) assignee. Method for treating premature ejaculation with a botulinum neurotoxin. US 8147848 B2. 2012.

[28] Simões Paço J, Jorge Pereira B. New therapeutic perspectives in premature ejaculation. Urology 2016; 88: 87-92. 


\section{حقن مادة البوتيولينم داخل البروستاتا لعلاج سرعة القذف: دراسة مستقبلية \\ طه ابو المجد عبدالمجيد'، '، احمد جلال الصياد'، عبدالملك سعيد طيب'، حسن محمد فارسي'، هثام

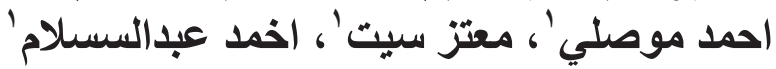

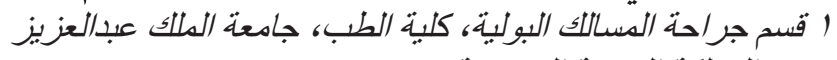

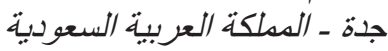 r قسم جراحة المسالك البولبية، كلبة الطب، البعة جامعة المنبا، مصر}

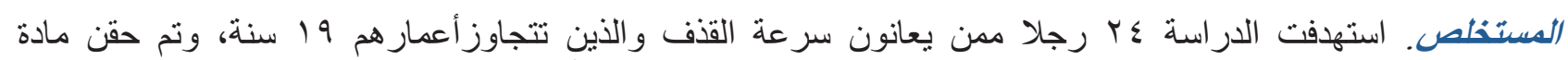

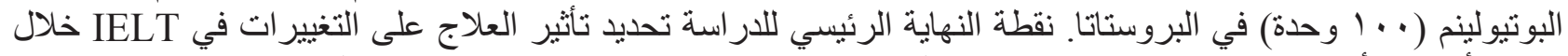

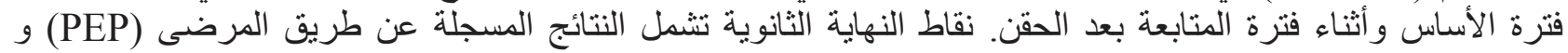
(PGI)

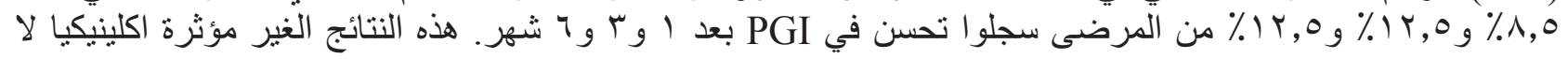

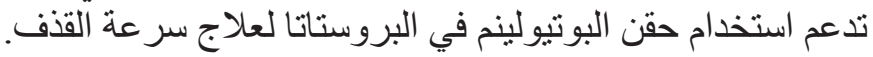

